\title{
The Dynamics of Spreading of Oils on Hydrophilic and Hydrophobic Surfaces Revisited Using Computational
} Fluid Dynamics

\author{
Badra Ali Talha ${ }^{1}$, Hocine Alla ${ }^{1}$, Somia Freifer ${ }^{1}$ and Thibault Roques-Carmes ${ }^{2,3}$ \\ 1. Laboratoire de Physique des Matériaux Et des Fluides, Université des Sciences et de la Technologie d'Oran, BP 1505 El \\ M'Naouar Bir el Djir 31000, Oran, Algeria \\ 2. Université de Lorraine, Laboratoire Réactions et Génie des Procédés, UMR 7274 CNRS, Nancy, F-54000, France \\ 3. CNRS, Laboratoire Réactions et Génie des Procédés, UMR 7274 CNRS, Nancy, F-54000, France
}

Received: November 21, 2013 / Accepted: December 11, 2013 / Published: December 25, 2013.

\begin{abstract}
The dynamics of drop spreading on horizontal smooth surfaces of different wettabilities is revisited using computational fluid dynamics (CFD). For this purpose, a recently developed CFD model, based on the volume of fluid technique (VOF), with piecewise linear interface calculations method (PLIC) for interface reconstruction, is generalized and applied to simulate the time evolution of spreading drops on solid surfaces (drop base radius and dynamic contact angle). The CFD simulations are quantitatively compared with previously published experimental results from other research groups. The influence of different factors, such as oils nature (silicone, mineral, peanut and coconut), viscosity (0.02-1 Pas $)$, drop volume (0.3-38 $\mu \mathrm{L})$ and type of surfaces (hydrophilic glass, stainless steel and hydrophobic glass) on the temporal evolution of the drop base radius and contact angle is investigated. For hydrophilic surfaces, the predictions of the CFD model agree remarkably well with the measurements. For hydrophobic surfaces, a small deviation between calculated and experimental results occurs because the model does not consider the partial slippage which can take place on hydrophobic materials. Despite neglecting this aspect, the simulations are found to capture the key features of drop spreading on hydrophobic surface. The fact that we obtain a good agreement between the proposed theory and the experimental results for a large range of oils and surfaces over five decades of time is a strong argument in favor of the model. The accuracy of the model demonstrates also that the influence of the surface wettabity (partial wetting and complete wetting) can be successfully simulated. The numerical results reproduce perfectly the spreading regimes which occur during the time course of the drop. The succession of two different regimes takes place in the following order: a hydrodynamic regime followed by a gravity regime.
\end{abstract}

Key words: Spreading dynamics, CFD simulation, oils, hydrophobic surfaces.

\section{Introduction}

The wetting of a solid by a liquid is a crucial part of many natural and industrial processes. Printing, painting, adhesion, lubrication, cleaning, coating, soldering, brazing and composite processing are few examples among the fields utilizing the phenomenon of wetting [1-4]. Owing to its importance in various

Corresponding author: Hocine Alla, professor, research fields: biphasic phenomenon-wetting and superspreading in solid surfaces-CFD-applied mathematics. E-mail: hocine.alla@partner.kit.edu. applications, the phenomena of drop spreading over solid surfaces have received a substantial attention in the literature [5-11]. However, the precise mechanism by which a liquid front advances across a solid remains only partially understood. Consequently, the present day ability to predict the dynamics of wetting and to model processes that are dependent on wetting is still significantly restricted [11].

In order to better understand the complex hydrodynamics of wetting it seems necessary to develop a numerical model. The numerical modeling 
of contact-line motion is difficult because of the widely differing length-scales involved in the problem. The main computer simulation methods includes computational fluid dynamics [12, 13], lattice Boltzmann [14, 15], and molecular dynamics [16, 17]. It appears that the available methods for wetting simulation display limitations and none of the above approaches can be used as general numerical solution for dynamic wetting problems. Ideally, one would combine the three methods within a synergistic multiscale procedure, using each of them wherever/whenever appropriate in different regions of the microflow [18]. The computational fluid dynamics based on the volume of fluid method (CFD/VOF) remains the most preferred method for the computations of drop impact and spreading where strong topological changes of interfaces occur [12, 13, 19-21]. This is a fixed-mesh method, in which the interface between immiscible fluids is modeled as the discontinuity in characteristic function (such as volume fraction). In a recent article, we developed a dynamic contact angle model into the VOF method to describe and simulate the dynamics of spreading drops previously deposited on a surface [22]. We studied the spreading of glycerol-water mixtures with and without surfactant on hydrophilic glass surfaces for a large range of viscosity $(0.02-1.15 \mathrm{~Pa} \cdot \mathrm{s})$, surface tension $(29-64 \mathrm{mN} / \mathrm{m})$ and drop volume $(8-60 \mu \mathrm{L})$. The simulations showed reasonably good agreement with the experimental data in conditions of partial wetting $[22,23]$. However, due to experimental limitations in our laboratory, no comparison was performed at very short time (lower than $1 \mathrm{~ms}$ ) and also at very long time (higher than $180 \mathrm{~s}$ ).

In addition, the previous numerical work was limited to a small number of liquids (water and glycerol-water mixtures) and substrate (hydrophilic glass). Numerical studies involving new substrates, more particularly hydrophobic substrates, and new kinds of liquids would be expected to be highly revealing. In line with this reasoning, the dynamics of drop spreading of several oils on surfaces of different wettabilities such as hydrophobic glass, stainless steel and hydrophilic glass is investigated. Different oils including silicone, mineral and vegetable oils (peanut and coconut) are used. The simulated results can be compared to the experimental data reported by Narayan Prabhu et al. [24] and Cazabat et al. [25]. The aim of this paper is to verify the validity of the model mentioned above, over several decades of time, for the different spreading regimes that are observed with a large range of surface wetting characteristics and liquid properties (partial or total wetting).

\section{Computational Fluid Dynamics Model}

We study the time evolution of a sessile liquid drop, which is initially put in contact with a flat horizontal solid surface (at time $t=0$ ), exposed to air in a nonequilibrium configuration and then evolves towards its equilibrium shape. The aim of the present work is to address the spreading in the capillary regime, where inertial effects are small. For that reason, the spreading is examined in situations where the drops are gently contacted with the surface (drop impact velocity $<<1 \mathrm{~m} / \mathrm{s}$ ). For the CFD model, the multiphase flow must be initialized so that the simulation starts from a stable initial position. Because it is impossible to specify the exact initial conditions corresponding to the experiments in the VOF simulations, all calculations are initiated with the experimental value of the initial radius.

We first introduce the solution domain and the computational grids. Three-dimensional modeling has been carried out by several researchers $[13,20,21]$ with results confirming that, in general, the $3 \mathrm{D}$ models are superior over the 2D models. However, 2D modeling still takes preference over 3D modeling due to the excessive computational time and expense that $3 \mathrm{D}$ geometry induces [26]. It appears that 3D modeling becomes necessary for particular configurations such as drop impacting onto a surface at high-impact velocities (>1 m/s) [19] and also the spreading of drop 
on inclined surfaces [13, 27]. Therefore, for low-impact velocities $(<1 \mathrm{~m} / \mathrm{s})$, an axisymmetric $2 \mathrm{D}$ domain was successfully used to carry out simulations of drop impact on horizontal surfaces [20]. In the same way, several 2D and 3D numerical tests performed to simulate the dynamics of spreading in the case of a drop previously deposited on horizontal surfaces have demonstrated the accuracy of the 2D model [22, 28]. In this study, a two dimensional modeling is constructed since we numerically follow the time evolution of a drop initially placed on a horizontal surface. The axisymmetric solution domain is shown in Fig. 1a. The computational grid is generated using GAMBIT. The two-dimensional computational domain is a rectangle with nominal dimensions of $10 \mathrm{~mm} \times 50 \mathrm{~mm}$ (Fig. 1b). The computational domain is mapped with structured square meshes of constant size $(\Delta x)$. A mesh size of $\Delta x$ $=5 \times 10^{-4} \mathrm{~mm}$ and a time step of $\Delta \mathrm{t}=10^{-5} \mathrm{~s}$ are used for all the simulations.

The volume of fluid approach (VOF), with piecewise linear interface calculations method (PLIC) for interface reconstruction, is applied to simulate the liquid/gas interface and also the drop spreading on the solid surface. A VOF interface tracking method is adopted to represent the fluid domain and track the evolution of the domain's free boundaries. Gas and liquid phases are considered to be incompressible, laminar and Newtonian fluids with constant values of viscosity and surface tension. The continuum surface force technique (CFS) is introduced to model surface forces and surface adhesion [29].

The two-fluid flow is modeled with the Navier-Stokes equation

$$
\frac{\partial \mathbf{u}}{\partial \mathrm{t}}+\nabla(\mathbf{u u})=-\frac{1}{\rho}\left(\nabla \mathrm{P}-\mu \nabla^{\mathbf{2}} \mathbf{u}\right)+\mathrm{g}+\frac{1}{\rho} \mathbf{F}_{\mathrm{SF}}(1)
$$

where, u represents the velocity vector, $\mathrm{P}$ the pressure, $\mu$ is the dynamic viscosity, $\rho$ is the density, $g$ is the gravitational acceleration, $\nabla$ is the standard spatial grad operator and $\mathrm{F}_{\mathrm{SF}}$ is the continuum surface force vector due to the surface tension.

The velocity field $u$ is subject to the incompressibility constraint

$$
\nabla \cdot \mathbf{u}=0
$$

This single set of flow equations is utilized throughout the domain and mixture properties as defined below are used.

The two fluids are immiscible (L: liquid and G: gas phase). Density and viscosity are constant in each phase but may be discontinuous at the interface. A scalar parameter of $\mathrm{F}$ is defined which identifies the fraction of the volume of each computational cell in the grid that is occupied by the liquid (Fig. 1a). The volume fraction of gas is by definition 1-F. Thus, F takes the value one in cells that contain only liquid and the value zero in cells that contain only air. A cell
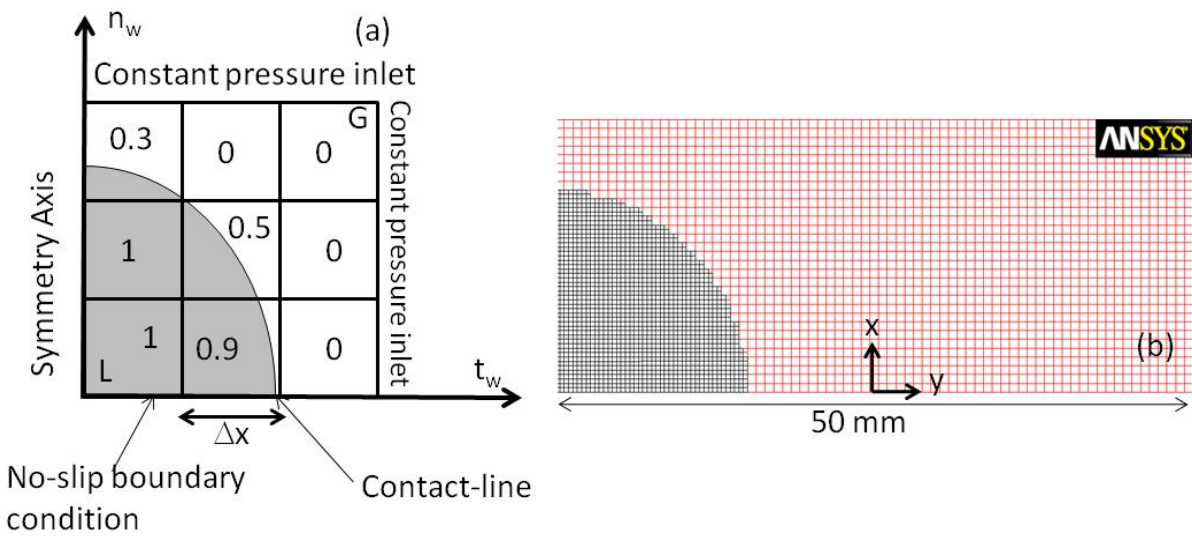

Fig. 1 Schematic diagram of the free-surface reconstructions: (a) Actual interface: the exact VOF color function for a circular arc over a square grid. The figure also contains the solution domain and boundary conditions and (b) Interface reconstruction with PLIC method. The number of cells is reduced compared to those used in the actual simulation for clarity reasons. 
that contains the gas/liquid interface would have an $\mathbf{F}$ value between zero and one. We use a volume fraction field $\mathbf{F}$ to represent and track the interface which is transported by the velocity field $\mathbf{u}$ :

$$
\frac{\partial \mathrm{F}}{\partial \mathrm{t}}+\mathbf{u} \cdot \nabla \mathbf{F}=0
$$

This equation allows for the calculation of the density $(\rho)$ and the dynamic viscosity $(\mu)$. In fact, the average values of density and viscosity of the mixture are interpolated by the formulas

$$
\begin{aligned}
& \rho=\rho_{L}+\left(\rho_{G}-\rho_{L}\right) \times F \\
& \mu=\mu_{L}+\left(\mu_{G}-\mu_{L}\right) \times F
\end{aligned}
$$

where, the subscripts $L$ and $G$ denote liquid and gas phases, respectively.

The surface tension model incorporated in FLUENT is the CSF model proposed by Brackbill et al. [29]. With this approach, the contribution of the surface tension force to the VOF calculation results in a source term in the momentum equation and is represented as

$$
\mathbf{F}_{\mathrm{SF}}=\gamma \frac{\rho \alpha \nabla \mathrm{F}}{\frac{1}{2}\left(\rho_{\mathrm{G}}+\rho_{\mathrm{L}}\right)}
$$

where, $\gamma$ is the liquid/gas surface tension and $\alpha$ the curvature of the interface.

The curvature of the interface can be written as

$$
\boldsymbol{\alpha}=\frac{\mathbf{1}}{|\mathbf{n}|}\left[\left(\frac{\mathbf{n}}{|\mathbf{n}|} \nabla\right)|\mathbf{n}|-(\nabla \mathbf{n})\right]
$$

The normal vector to the surface $\mathbf{n}$ is evaluated in the cells that contain the liquid/gas interface and requires the knowledge of the amount of volume of fluid present in the cell.

Surface reconstruction becomes necessary for the determination of the flux of liquid from one cell to the next, and surface curvatures when the surface tension model is activated. For this purpose, the VOF algorithm updates the volume fraction field $\mathrm{F}$ given the fixed grid, the velocity field $\mathrm{u}$ and the field $\mathrm{F}$ at the previous step. In this approach, a straight-line segment approximates an interface within a computational cell.
The interface is considered as a continuous piecewise smooth line, and the problem of its reconstruction is that of finding the approximation to the section of the interface in each cell, by knowing only the volume fraction $\mathrm{F}$ in that cell and in the neighboring cells. In other words, the VOF technique based on piecewise linear interface calculation (PLIC) fits the interface with piecewise linear segments $[30,31]$. Fig. 1a reproduces the exact color VOF function for a smooth circular arc. Fig. $1 \mathrm{~b}$ displays an interface reconstructed with VOF/PLIC. Note that the number of cells depicted in the figure is reduced for clarity reasons. Because $\mathrm{F}$ in Eq. (3) is not a continuous function, the VOF/PLIC algorithm is divided into two parts: a reconstruction Step (1) and a propagation Step (2). The reconstruction Step (1) leads to the determination of the orientation of the segment. This is equivalent to the determination of the unit normal vector $\mathrm{n}$ to the segment. The normal vector $\mathrm{n}$ and the volume fraction $\mathrm{F}$ then uniquely determine the straight line. The propagation Step (2) is performed using a fractional step or an operator split method, which updates the volume fraction $F$ by advecting the interface along one spatial direction at a time. The intermediate $\mathrm{F}$ values are estimated during this process, and the final $\mathrm{F}$ field is obtained after advection of the interface along all coordinate directions.

Wall adhesion is introduced in the model through the contact angle. For fluids exhibiting a nonzero contact angle, the presence of wall also affects the surface normal and therefore wall adhesion forces are included in the present work. The wall adhesion is modeled in a manner similar to that of the surface tension in the case of a gas/liquid interface [29], except that the unit normal $\mathrm{n}$ in this case is evaluated using the dynamic contact angle $\theta_{\mathrm{d}}$ according to

$$
\mathbf{n}=\mathbf{n}_{\mathbf{w}} \times \cos \theta_{d}+\mathbf{t}_{\mathbf{w}} \times \sin \theta_{d}
$$

where $\mathbf{n}_{\mathbf{w}}$ and $\mathbf{t}_{\mathbf{w}}$ are the unit vector normal and tangential to the wall (Fig. 1a), respectively, and $\theta_{d}$ is the dynamic contact angle at the wall. Surface normal 
obtained from one cell above the wall and near the wall (from Eq. (8)) are employed to obtain the body surface force from surface tension. Several models describe the evolution of the contact angle of the fluid. One approach is to assign a constant value to the equilibrium contact angle $\theta$ neglecting the contact angle hysteresis. This is also referred to as a static contact angle model. An alternative way is the application of a varying value of the contact angle, which depends on instantaneous flow parameters. These models are called dynamic contact angle models. One of the most recent and accurate ones is the Kistler model [32], which estimates the dynamic contact angle $\theta_{\mathrm{d}}$ using the Hoffman function $\mathrm{f}_{\text {Hoff }}$ as follows

$$
\theta_{\mathrm{d}}=\mathrm{f}_{\text {Hoff }}\left\lfloor\mathrm{Ca}+\mathrm{f}_{\text {Hoff }}^{-1}(\theta)\right\rfloor
$$

where, $\theta$ is the equilibrium contact angle and $\mathrm{f}_{\text {Hoff }}^{-1}$ the inverse of the function $\mathrm{f}_{\text {Hoff }}$ which is defined as

$$
\begin{aligned}
& \mathrm{f}_{\text {Hoff }}(\mathrm{x})= \\
& \arccos \left[1-2 \tanh \left(5.16\left(\frac{\mathrm{x}}{1+1.31 \mathrm{x}^{0.99}}\right)^{0.706}\right)\right]
\end{aligned}
$$

The capillary number $\mathrm{Ca}$ is defined as $\mathrm{Ca}=\left(\mathrm{U}_{\mathrm{cl}} \times\right.$ $\mu) / \gamma$, where $U_{\mathrm{cl}}, \mu$ and $\gamma$ are the spreading velocity of the contact-line, the dynamic viscosity of the liquid and the surface tension of the liquid and gas phase, respectively. Eq. (9) shows that the dynamic contact angle $\theta_{\mathrm{d}}$ depends on the capillary number of the contact-line and requires the input of an equilibrium contact angle. For the present dynamic model, the Kistler approach is adopted with limiting equilibrium contact angles measured experimentally. To this extent, we use the experimental data reported by Narayan Prabhu et al. [24] and Cazabat and Cohen Stuart [25].

The boundary conditions are given in Fig. 1a. Along the symmetry axis (x-axis in Fig. 1b) a symmetric boundary condition is imposed in which normal velocity and normal gradients are set to zero, i.e. $\mathrm{u}=0$ and $\partial u / \partial x=0$. A no-slip boundary condition is introduced at the wall where all the components of velocity are set to zero (Fig. 1a). Although the no-slip boundary condition (zero velocity on wall) is implemented at the wall boundary, the gas/liquid/solid contact-line moves along the wall, presenting a kind of singularity. Because velocity profiles at the other two planes (other than symmetry and wall planes) of the solution domain are not known, a constant pressure boundary condition is used at these planes (Fig. 1a). However, in the numerical implementation of the VOF model, the solid wall boundaries coincided with the cell boundaries. The no-slip boundary condition is implemented by setting the velocity at such cell boundaries (faces) to zero. The velocities at the cell center or cell faces other than those adjacent to the solid surface are not zero. Such nonzero velocities influence the volume fraction field and thus the position of the interfaces. Thus, such implementation achieves movement of the gas/liquid/solid contact-line despite specifying a no-slip boundary condition at the solid surface.

The model equations given above are solved using the commercial flow solver Ansys Fluent 12.0.16. The spatial derivatives in Eqs. (1) and (3) are discretized using the quadratic upwind interpolation for convective kinetics system (QUICK) scheme while the temporal derivatives are discretized using a first-order implicit method. The pressure implicit with splitting of operator (PISO) scheme is applied for the pressure velocity coupling in the momentum equation.

\section{Results and Discussion}

\subsection{Spreading of Vegetable and Mineral Oils on Stainless Steel}

As a first configuration, we study the drop dynamics of vegetable and mineral oils on stainless steel substrates. On the one hand, this system allows us to analyze the spreading at very short time in conditions of partial wetting. On the other hand, it becomes possible to evaluate the CFD scheme using the dynamic contact angle model. The experimental results were previously reported by Narayan Prabhu and coworkers [24]. They used commercially available 
vegetable (peanut and coconut) and mineral oils.

In the simulations, a circular liquid drop which is initially put onto a solid surface in a nonequilibrium configuration is considered. The simulated spreading of the drop is represented in the form of contours of liquid phase volume fraction. The calculated drop shape at different time for coconut and mineral oils are reported in Fig. 2. The two drops display a similar behavior. After the impact on the surface, the drop starts from an initial position with a contact angle of $67^{\circ}$ and $97^{\circ}$ for coconut and mineral oils, respectively. Then, the drop begins to spread and the contact angle reduces within some milliseconds. During the wetting, the drop height decreases while the drop radius increases. This process continues until the drop reaches the equilibrium position. From the figure, the temporal evolution of the drop base radius and contact angle becomes observable. The numerical results can be also compared with the experimental time sequence photographs under the same experimental conditions [24]. The temporal evolutions of the shape of the drops are very similar to those presented in Ref. [24]. The proposed model satisfactorily describes the key qualitative features observed in the experiments.

We check the validity of the model by comparing the calculated and experimental dynamic contact angles as a function of time. The results are shown in Fig. 3 for a variety of oils. As expected, the calculated dynamic contact angle decreases with the time for all the oils. The oil starts spreading rapidly with a relatively high velocity. This results in a sharp diminution of the contact angle. However, within a very short period of time, the spreading rate reduces significantly to almost zero indicating the stabilization of the contact angle. This is due to the attainment of the equilibrium between the various surface forces under action. Further relaxation of the contact angle becomes negligible. The contact angle reaches an equilibrium value significantly higher than 0 (ca. $30-50^{\circ}$ ). This means that the oils do not wet the solid completely and confirms that we discuss the motion of (a) Coconut oil

(b) Mineral oil

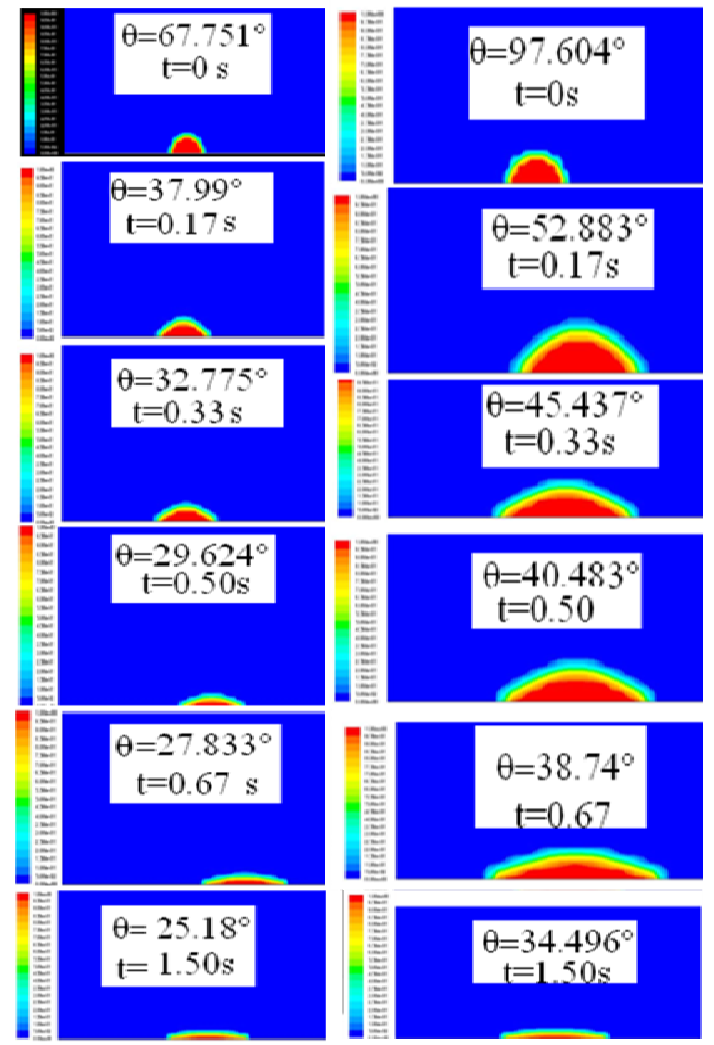

Fig. 2 Simulated time sequence snapshots of a drop spreading on stainless steel substrate for: (a) Coconut oil $(\gamma=29.4 \mathrm{mN} / \mathrm{m}, \mu=0.03$ Pa s), (b) Mineral oil $(\gamma=30.0$ $\mathrm{mN} / \mathrm{m}, \mu=0.08$ Pa s).

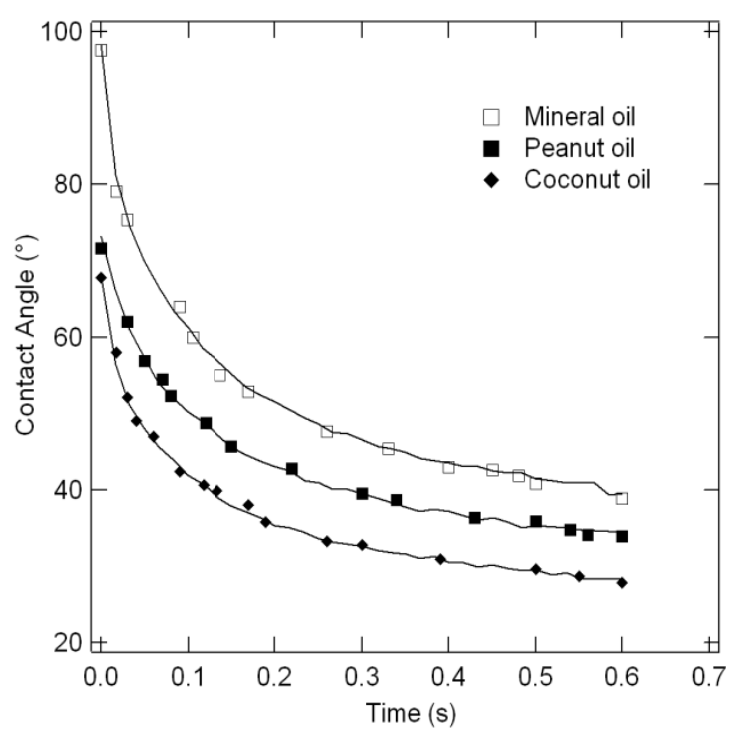

Fig. 3 Comparison of the experimental (symbols, from Narayan Prabhu et al. [24]) and calculated (lines) dynamic contact angle of various oils on stainless steel substrate as a function of time. Parameters used in calculations: Mineral oil: $\gamma=30 \mathrm{mN} / \mathrm{m}, \mu=0.08$ Pa s; Peanut oil: $\gamma=35.6 \mathrm{mN} / \mathrm{m}, \mu$ $=0.06$ Pa s; Coconut oil: $\gamma=29.4 \mathrm{mN} / \mathrm{m}, \mu=0.03$ Pa s. 
the contact-line in conditions of partial wetting. A very good agreement between the model and experimental data is found since the CFD approach accounts for almost all data points. Both the final contact angle as well as the temporal evolution of the wetting is well described. It is important to note that the numerical simulations reproduce quantitatively the experimentally observed drop behavior, only when we use the dynamic contact angle model. If we apply an equilibrium contact angle in the CFD approach (instead of the dynamic contact angle), the predicted time dependence of drop behavior is poorly reproduced (result not shown). This means that precise dynamic contact angle modeling plays an important role and is pertinent in the simulation of drop spreading. Similar observations were made by other groups [21, 33-35]. In the following, all calculations will be done using this construction.

The time evolution of the drop base radius is studied to monitor the ability of the model to reproduce the experimental spreading. The experimental data are compared with calculations from the CFD model (Fig. 4). The radius increases sharply with the time for all the series and then attains a plateau. The figure indicates that the model is able to satisfactorily describe the temporal evolution of the drop base radius since the simulation and experimental results show a relatively good agreement. Some minor differences can be recognized. It may be noted that VOF simulations, at present, are unable to account for microscopic surface characteristics such as surface heterogeneity or contamination. It was observed from the experimental images depicted in Ref. [24] that the stainless steel surfaces were not perfectly smooth and exhibited a slight surface heterogeneity. It is established, and experimentally verified, that microscopic factors such as surface heterogeneity and surface roughness have the potential to affect the drop dynamics $[5,6,36,37]$. We then consider that the observed small disagreement between experimental and simulated results comes mainly from the surface heterogeneity and roughness. As expected the oil viscosity plays a role in the speed of the spreading of the oil drop. As the oil viscosity increases the radius curve shifts to higher time. All the curves do not plateau at the same value and the final radius with coconut oil is the highest. Coconut oil shows rapid wetting behavior while the kinetics of spreading of peanut and mineral oils are slower. The viscosity values for these oils are significantly different. Coconut oil has the lowest viscosity $(\mu=0.03 \mathrm{~Pa} \cdot \mathrm{s})$. On the contrary, viscosity of mineral oil $(\mu=0.08 \mathrm{~Pa} \cdot \mathrm{s})$ is higher while peanut oil shows intermediate viscosity ( $\mu=0.06 \mathrm{~Pa} \cdot \mathrm{s})$. Consequently, coconut oil exhibits accelerated wetting speed owing to its lower viscosity as compared to mineral oil while peanut oil shows intermediate behavior. This establishes that the theory takes into account the change of liquid viscosity. The CFD estimation of the evolution of the drop radius for different viscosities is necessary since the dynamics of spreading is modified by the liquid viscosity. The accuracy of the model results confirms that the influence of the liquid viscosity is satisfactorily described.

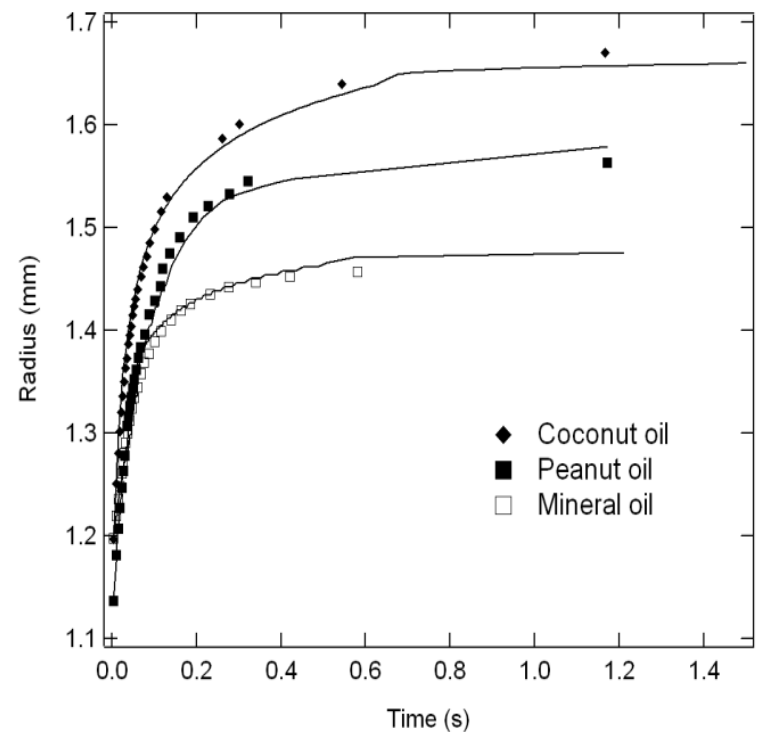

Fig. 4 Comparison of the experimental (symbols, from Narayan Prabhu et al. [24]) and calculated (lines) time evolution of the drop radius of various oils on stainless steel substrate. Parameters used in calculations: mineral oil: $\gamma=$ $30 \mathrm{mN} / \mathrm{m}, \mu=0.08$ Pa s; peanut oil: $\gamma=35.6 \mathrm{mN} / \mathrm{m}, \mu=0.06$ Pa s; Coconut oil: $\gamma=29.4 \mathrm{mN} / \mathrm{m}, \mu=0.03$ Pa s. 
To summarize, the different wetting properties including the drop base radius and the dynamic contact angle can be successfully simulated at very short time. This is a quite interesting result since the dynamic wetting behavior is significantly affected during the initial stage of spreading. Both, the contact angle relaxation and the increase of the drop base radius are sharp. The model captures the essential physics of the initial stage of wetting when the oils spread rapidly with a relatively high velocity.

\subsection{Spreading of Silicone oil on Hydrophilic Glass}

To test the validity of the model over a longer period of time and in condition of total wetting, additional data corresponding to spreading experiments performed with silicone oils on hydrophilic glass surfaces are compared to the model. The experimental data were previously obtained by Cazabat and Cohen Stuart [25]. The hydrophilic surfaces were microscope glass slides. Two silicone oils (poly(dimethylsiloxane), methyl terminated) of viscosities of 0.02 and $1 \mathrm{~Pa} \mathrm{~s}$, respectively, were used. It was experimentally observed that the two silicone oils completely wet the hydrophilic glass surfaces and that the contact-line was preceded by a thin precursor film [25].

The calculated shape of the drop at different time for two drop volumes is given in Fig. 5. It can be seen that each drop has a similar evolution. The drop spreads slowly and it takes some minutes to observe a significant drop of the contact angle. During the spreading, the drop height is reduced while the drop radius increases. This phenomenon pertains until the drop reaches the equilibrium position which is attained after 500-600 s. It is very attractive to note that the equilibrium shape of the drop is close to the final "pancake" which is generally expected in the case of complete wetting $[5,6]$.

In Fig. 6, the experimental and simulated radiuses are plotted together versus time for several drop volumes and viscosities. Each curve shows a similar (a) $\mathrm{V}=0.8 \mu \mathrm{L}$
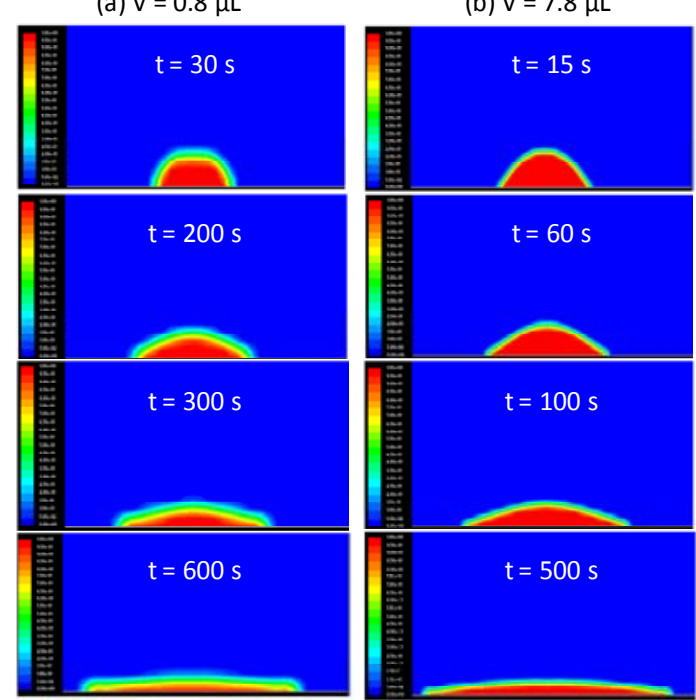

Fig. 5 Simulated time sequence snapshots of a drop of silicone oil spreading on hydrophilic glass surface for two drop volumes. Parameters used in calculations: $\gamma=24 \mathrm{mN} / \mathrm{m}$ and $\mu=0.02$ Pa s.
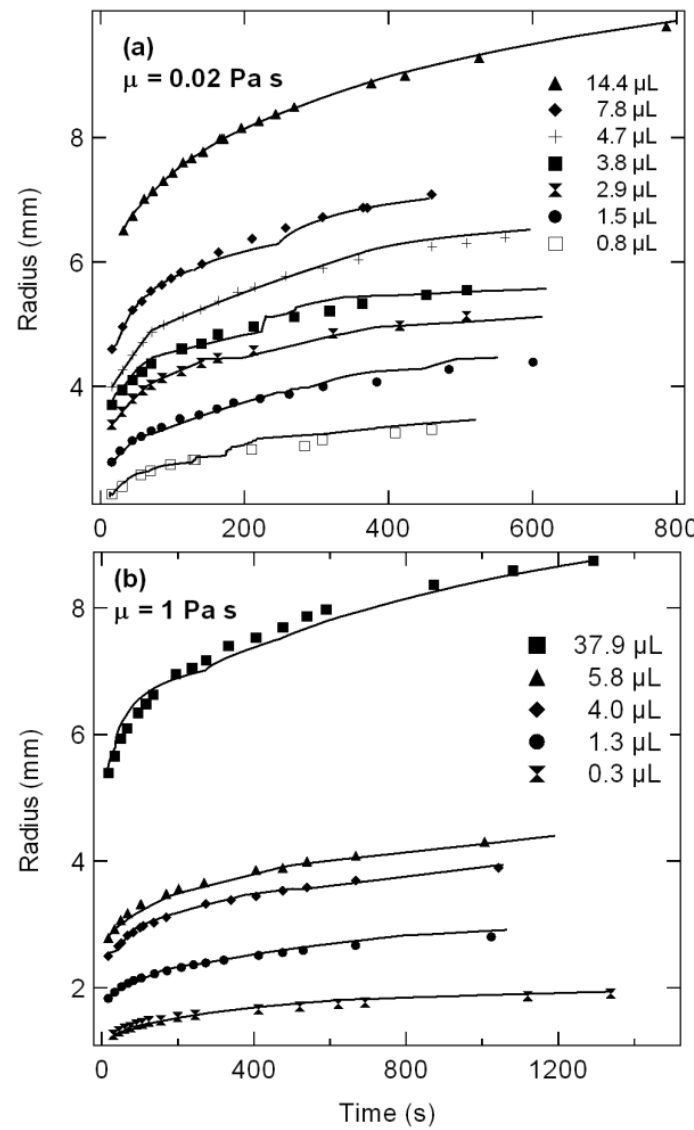

Fig. 6 Comparison of the experimental (symbols, from Cazabat and Cohen Stuart [25]) and calculated (lines) time evolution of the drop radius of silicone oils for different drop volumes on hydrophilic glass surface. Parameters used in calculations: $\gamma=24 \mathrm{mN} / \mathrm{m}$. (a) $\mu=0.02$ Pa.s, (b) $\mu=1$ Pa.s. 
trend. The radius increases gradually with the time for all the series. The time evolution of the drop depends on the drop volume. The wetting velocity increases with the drop size since the curves become somewhat steeper. In addition, the final radius increases with the drop volume. The calculated results compare well with the experimental data over a considerable temporal range (up to 1,300 s). The small deviation visible here for drop volumes of $37.9,7.8$ and $3.7 \mu \mathrm{L}$ is attributed to the temporal resolution. However the maximum difference between the values obtained by both methods is around $0.05 \mathrm{~mm}$ which is actually within the margin of error of the radius measurements [23, 25]. The accuracy of the model results emphasizes that the theory remains valid for a large range of drop volume $(0.3-38 \mu \mathrm{L}$ ) during a very long time (up to $1,300 \mathrm{~s}$ ). This is a very important result since the nature of the spreading (gravity spreading or capillary spreading) is significantly affected by the drop volume. In addition, we have shown the validity of the model in the case of complete wetting. This demonstrates that the CFD approach takes into account the energy dissipation in the precursor film $[5,6]$.

It was previously observed that several spreading regimes occurred during the time course of a drop. The succession of a hydrodynamic regime $(\mathrm{R}(\mathrm{t}) \approx$ $t^{1 / 10}$, where $R$ is the drop radius and $t$ the time) and lastly a gravity regime $\left(\mathrm{R}(\mathrm{t}) \approx \mathrm{t}^{1 / 8}\right)$ was experimentally observed $[25,38-41]$. To validate the succession of these 2 regimes, the calculated drop radius has been plotted as a function of time on a log-log scale (Fig. 7). The results presented in the figure are fairly representative for the other results. In all cases, a similar evolution can be observed. A short early-time stage is characterized by a non-linear curve. It is followed by 2 successive linear sections. These linear sections that correspond quite obviously each to a specific spreading regime are linked one another by a non-linear stage corresponding to an intermediate situation. The linear increase of the drop radius on a log-log scale means that the corresponding spreading regime can be described by a power law of the form $\mathrm{R}$ $=\mathrm{K} \times \mathrm{t}^{\mathrm{m}}$, where $\mathrm{m}$ and $\mathrm{K}$ are the two constant parameters depending on the experimental conditions but also on the time range [23]. For each section, the $\mathrm{m}_{\mathrm{i}}$ and $K_{i}$ parameters are optimized, the subscript $\mathrm{i}(\mathrm{i}=1-2)$ referring to the first and second linear sections. All the results are listed in Tables 1 and 2. The power law exponents do not vary with the viscosity and the drop volume. The average $m_{1}$ value equals $0.104 \pm 0.004$ while $\mathrm{m}_{2}=0.124 \pm 0.003$. In the first regime, the power law exponents $\mathrm{m}_{1}$ correspond well to theoretically predicted exponent by the hydrodynamic theory $(\mathrm{m}=1 / 10)$. In the second regime, the power laws $\mathrm{m}_{2}$ are in fair agreement with the exponent corresponding to a gravity regime $(\mathrm{m}=1 / 8)$. The model

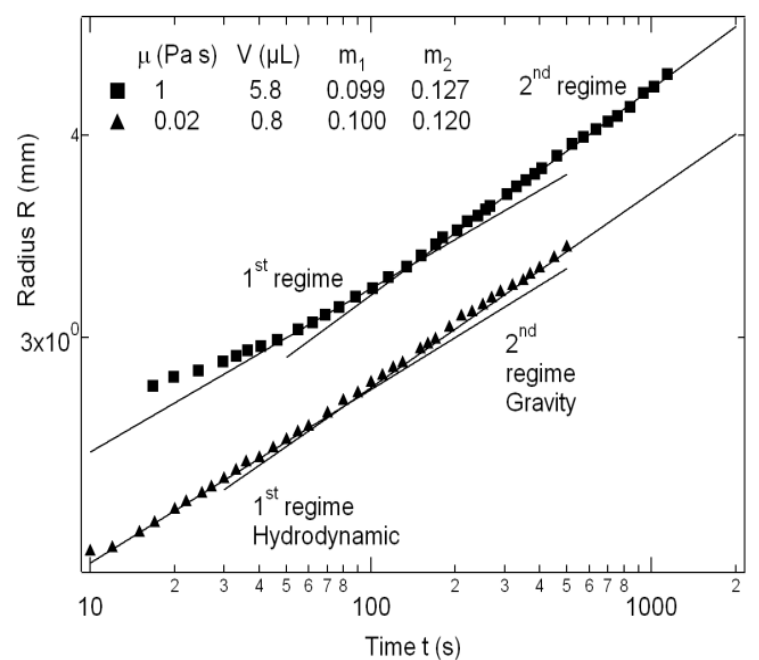

Fig. 7 Calculated time evolution of the drop radius of silicone oils on hydrophilic glass surface on a log-log scale. Two distinguished parts of the curve are fit by linear regression (lines). Parameter used in calculations: $\gamma=\mathbf{2 4}$ $\mathbf{m N} / \mathbf{m}$.

Table 1 Influence of the drop volume (V) on the calculated spreading parameters of silicone oils $(\mu=1.00 \mathrm{~Pa} \cdot \mathrm{s})$ on hydrophilic glass surface.

\begin{tabular}{cllll}
\hline \multirow{2}{*}{$\mathrm{V}(\mu \mathrm{L})$} & \multicolumn{2}{c}{ 1st regime } & \multicolumn{2}{c}{ 2nd regime } \\
\cline { 2 - 5 } & $\mathrm{m}_{1}$ & $\mathrm{~K}\left(\mathrm{~mm} \cdot \mathrm{s}^{-\mathrm{m}}\right)$ & $\mathrm{m}_{2}$ & $\mathrm{~K}\left(\mathrm{~mm} \cdot \mathrm{s}^{-\mathrm{m}}\right)$ \\
\hline 37.9 & 0.097 & 4.17 & 0.124 & 3.84 \\
5.8 & 0.099 & 2.18 & 0.127 & 1.77 \\
4.0 & 0.100 & 1.81 & 0.122 & 1.71 \\
1.3 & 0.103 & 1.34 & 0.122 & 1.15 \\
0.3 & 0.109 & 0.87 & 0.118 & 0.89 \\
\hline
\end{tabular}


reproduces the experimentally determined hydrodynamic and gravity regimes. It is also important to note that the model successfully describes the non-linear stages (in a log-log scale) corresponding to intermediate situation between the spreading regimes. The CFD quantitative estimation of the evolution of the drop radius in the intermediate state is necessary since it is not provided by the classical theories.

\subsection{Spreading of Silicone Oil on Hydrophobic Surface}

To estimate the effect of the surface hydrophobicity, the numerical results are compared with experiments performed with silicone oils on hydrophobic glass surfaces. The experimental data were previously reported by Cazabat and Cohen Stuart [25]. Smooth, hydrophobic surfaces were obtained by reaction of the glass slides with hexadecanol in the vapor phase.

The simulated and experimental time evolution of the drop radius for several drop volumes is presented in Fig. 8. For drop volume less than or equal to $1.5 \mu \mathrm{L}$, the calculated results compare perfectly well with the experimental data. However, for drop volume larger than $1.5 \mu \mathrm{L}$, the difference between the numerical and experimental results becomes significant. In general simulations overpredicted the radiuses compared to those observed in the experiments since the simulated

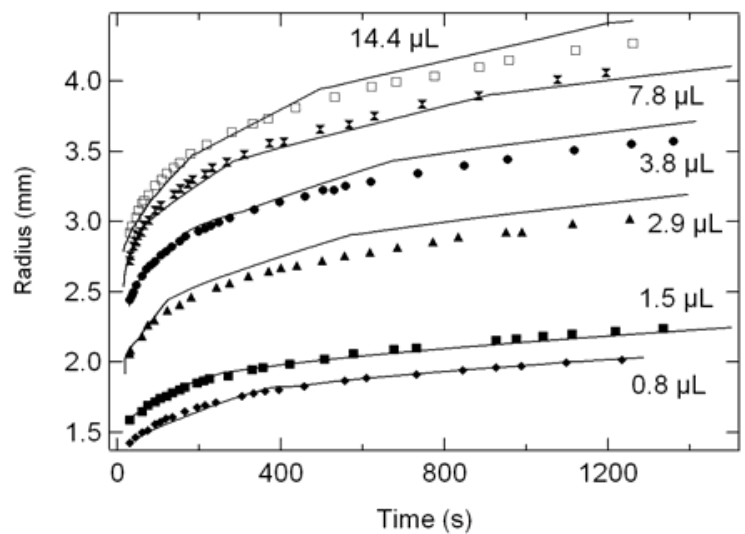

Fig. 8 Comparison of the experimental (symbols, from Cazabat and Cohen Stuart [25]) and calculated (lines) time evolution of the drop radius of silicone oils for different drop volumes on hydrophobic glass surface. Parameters used in calculations: $\gamma=24 \mathrm{mN} / \mathrm{m}$ and $\mu=1 \mathrm{~Pa} \cdot \mathrm{s}$. radiuses are much larger than that observed experimentally. The deviation is attributed to the no-slip boundary condition used at the surface. However, in recent years, it has become clear that the no-slip boundary condition at the solid/liquid interface is only valid for smooth hydrophilic surfaces [42]. Thus, the hydrophobicity of smooth surfaces could induce a partial slippage [42-44]. In this case, the slip length can be of the order of tens of nanometers [42]. However, this aspect has been neglected in the present study and could explain the discrepancy between the experimental and calculated results. Therefore, in spite of ignoring the slippage, the VOF method can capture the spreading behavior on hydrophobic materials, and it can be inferred that the experimentally and numerically observed drop raduises are in acceptable agreement. It should be also noted that the CFD approach has better predictive accuracy for the small drop volume. This confirms the validity of the model with hydrophobic surface.

On hydrophobic surfaces, the succession of a hydrodynamic regime $\left(\mathrm{R}(\mathrm{t}) \approx \mathrm{t}^{1 / 10}\right)$ followed by a gravity regime $\left(\mathrm{R}(\mathrm{t}) \approx \mathrm{t}^{1 / 8}\right)$ was experimentally demonstrated $[25,45]$. The possible apparition of these 2 regimes can be analyzed by plotting the calculated drop radius against time on a log-log scale (not shown). The spreading process goes through a combination of two different linear regimes regardless of the drop volume. Table 3 shows the influence of the drop volume on the values of the power law exponents and the pre-exponential factor $\mathrm{K}$. The slopes $\mathrm{m}_{1}$ and $\mathrm{m}_{2}$ do not vary with the drop volume. The average $m_{1}$ value becomes $0.105 \pm 0.004$ and $m_{2}=0.124 \pm 0.003$. In the first regime, the power laws correspond well to theoretically predicted exponent for the hydrodynamic regime $(\mathrm{m}=1 / 10)$. For the second regime, the power law exponents refer to a gravity regime $(\mathrm{m}=1 / 8)$. The succession of the hydrodynamic and gravity regimes can be accurately reproduced by means of the model. The CFD approach is able to specify the kinetic regime associated with different mode of energy 
Table 2 Influence of the drop volume (V) on the calculated spreading parameters of silicone oils $(\mu=0.02$ Pa·s) on hydrophilic glass surface.

\begin{tabular}{cllll}
\hline $\mathrm{V}(\mu \mathrm{L})$ & \multicolumn{2}{c}{ 1st regime } & \multicolumn{2}{c}{ 1st regime } \\
\cline { 2 - 5 } & $\mathrm{m}_{1}$ & $\mathrm{~K}\left(\mathrm{~mm} \cdot \mathrm{s}^{-\mathrm{m}}\right)$ & $\mathrm{m}_{2}$ & $\mathrm{~K}\left(\mathrm{~mm} \cdot \mathrm{s}^{-\mathrm{m}}\right)$ \\
\hline 14.4 & 0.105 & 4.51 & 0.121 & 4.05 \\
7.8 & 0.111 & 3.26 & 0.122 & 3.08 \\
3.8 & 0.107 & 2.72 & 0.128 & 2.49 \\
2.9 & 0.108 & 2.51 & 0.125 & 2.45 \\
1.5 & 0.107 & 2.10 & 0.121 & 1.63 \\
0.8 & 0.100 & 1.70 & 0.120 & 1.60 \\
\hline
\end{tabular}

Table 3 Influence of the drop volume (V) on the calculated spreading parameters of silicone oils $(\mu=1.00 \mathrm{~Pa} \cdot \mathrm{s})$ on hydrophobic glass surface.

\begin{tabular}{cllll}
\hline \multirow{2}{*}{$(\mu \mathrm{L})$} & \multicolumn{2}{c}{ 1st regime } & \multicolumn{2}{c}{ 1st regime } \\
\cline { 2 - 5 } & $\mathrm{m}_{1}$ & $\mathrm{~K}\left(\mathrm{~mm} \cdot \mathrm{s}^{-\mathrm{m}}\right)$ & $\mathrm{m}_{2}$ & $\mathrm{~K}\left(\mathrm{~mm} \cdot \mathrm{s}^{-\mathrm{m}}\right)$ \\
\hline 14.4 & 0.099 & 1.98 & 0.128 & 1.73 \\
7.8 & 0.101 & 1.94 & 0.122 & 1.70 \\
3.8 & 0.098 & 1.66 & 0.123 & 1.52 \\
2.9 & 0.109 & 1.42 & 0.129 & 1.35 \\
1.5 & 0.099 & 1.17 & 0.120 & 1.01 \\
0.8 & 0.097 & 1.01 & 0.123 & 0.96 \\
\hline
\end{tabular}

dissipation during the drop shape transformation (gravity and hydrodynamic effects) and also the time window in which each particular regime hold preferentially.

\section{Conclusions}

The aim of this study was to investigate the dynamics of drop spreading on surfaces of different wettabilities (hydrophilic and hydrophobic surfaces), over several decades of time, using a computational fluid dynamics model (CFD). The model applies the volume of fluid method (VOF) and has been implemented to simulate the time evolution of the drop base radius and contact angle to the case of a drop previously deposited on a surface. The simulations take into account the surface tension and wall adhesion phenomenon. We also introduce a dynamic contact angle model into the VOF method. The only adjustable parameter is the initial conditions of simulation (initial drop radius and initial contact angle) which has to be identical to those of the experiments.

The CFD predictions have been validated using experimental data reported by other research groups. The oils nature (silicone, mineral, peanut and coconut), viscosity, drop volume and type of surfaces (hydrophilic glass, stainless steel and hydrophobic glass) have been varied. For hydrophilic surfaces, the results indicate that the predictions of the model agree remarkably well with the measurements. The simulations give much better agreement when dynamic contact angle is used instead of an equilibrium value. For hydrophobic surfaces, the CFD simulations show a small discrepancy with the experimental data. The deviation is attributed to the no-slip boundary condition used at the surface while the surface hydrophobicity could induce a partial slippage. Despite neglecting this aspect, the proposed model captures reasonably well the key features of oils spreading on hydrophobic materials. The calculations performed confirm that the oil viscosity and the drop volume play a role in the speed of the drop motion.

The numerical results reproduce perfectly the several spreading regimes which occur during the time course of the drop. The succession of a hydrodynamic regime $\left(\mathrm{R}(\mathrm{t}) \approx \mathrm{t}^{1 / 10}\right)$ and lastly a gravity regime $(\mathrm{R}(\mathrm{t})$ $\left.\approx t^{1 / 8}\right)$ is demonstrated. The CFD approach is able to specify the kinetic regime associated with the different mode of energy dissipation during the drop shape transformation (gravity and hydrodynamic effects) and also the time window in which each particular regime hold preferentially.

Theoretical results obtained in this investigation have been quite encouraging. We have shown that the calculated results compare well with the experimental data for a large range of oils and surfaces. The fact that we obtain a good agreement between the proposed theory and the experimental values over five decades of time is a strong argument in favor of the model. We also demonstrate that the influence of the surface wettability (partial wetting and complete wetting) can be successfully simulated. Further work will extend the present work to address the modeling of the 
dynamics of drop spreading on rough surfaces. This will be useful since most practical solid surfaces are rough to some extent, and for some applications of wetting, roughness of the surface may even be essential.

\section{References}

[1] G. Kumar, K.N. Prabhu, Review of non-reactive and reactive wetting of liquids on surfaces, Adv. Colloid Interface Sci. 133 (2007) 61-89.

[2] T. Roques-Carmes, F. Aldeek, L. Balan, S. Corbel, R. Schneider, Aqueous dispersions of core/shell CdSe/CdS quantum dots as nanofluids for electrowetting, Colloids Surf. A 377 (2011) 269-277.

[3] M. von Bahr, F. Tiberg, V. Yaminsky, Spreading dynamics of liquids and surfactants solutions on partially wettable hydrophobic, Sci. 39 (1992) 1-11.

[4] T. Roques-Carmes, A. Gigante, J.M. Commenge, S. Corbel, Use of surfactants to reduce the driving voltage of switchable optical elements based on electrowetting, Langmuir 25 (2009) 12771-12779.

[5] P.G. de Gennes, Wetting: statics and dynamics, Rev. Mod. Phys. 57 (1985) 827-863.

[6] D. Bonn, J. Eggers, J. Indekeu, J. Meunier, E. Rolley, Wetting and spreading, Rev. Mod. Phys. 81 (2009) 739-805.

[7] F. Brochard-Wyart, P.G. de Gennes, Dynamics of partial wetting, Adv. Colloid Interface Sci. 39 (1992) 1-11.

[8] A.M. Cazabat, S. Gerdes, M.P. Valignat, S. Villette, Dynamics of wetting: from theory to experiment, Interface Sci. 5 (1997) 129-139.

[9] M.J. de Ruijter, J. De Coninck, G. Oshanin, Droplet spreading: Partial wetting regime revisited, Langmuir 15 (1999) 2209-2216.

[10] M.J. de Ruijter, M. Charlot, M. Voué, J. De Coninck, Experimental evidence of several time scales in drop spreading, Langmuir 16 (2000) 2363-2368.

[11] T.D. Blake, The physics of moving wetting lines, J. Colloid Interface Sci. 299 (2006) 1-13.

[12] C.W. Hirt, B.D. Nichols, Volume of Fluid (VOF) method for the dynamics of free boundaries, J. Comput. Phys. 39 (1981) 201-225.

[13] S.F. Lunkad, V.V. Buwa, K.D.P. Nigam, Numerical simulations of drop spreading on horizontal and inclined Surfaces: Dynamic contact angle approach, Chem. Eng. Sci. 62 (2007) 7214-7224.

[14] J. Harting, C. Kunert, H.J. Herrmann, Lattice Boltzmann simulations of apparent slip in hydrophobic microchannels, Euro. Phys. Lett. 75 (2006) 328-334.

[15] S. Mukherjee, J. Abraham, Investigations of drop impact on dry walls with a Lattice-Boltzmann model, J. Colloid Interface Sci. 312 (2007) 341-354.

[16] G. Martic, F. Gentner, D. Seveno, D. Coulon, J. De Coninck, T.D. Blake, A molecular dynamics simulation of capillary imbibition, Langmuir 18 (2002) 7971-7976.

[17] F. Gentner, G. Ogonowski, J. De Coninck, Forced wetting dynamics: a molecular dynamics study, Langmuir 19 (2003) 3996-4003.

[18] S. Chibbaro, E. Costa, D.I. Dimitrov, F. Diotallevi, A. Milchev, D. Palmieri, et al., Capillary filling in microchannels with wall corrugations: a comparative study of the concus-finn criterion by continuum, kinetic, and atomistic approaches, Langmuir 25 (2009) 12653-12660.

[19] P.R. Gunjal, V.V. Ranade, R.V. Chaudhari, Dynamics of drop impact on solid surface: Experiments and VOF simulations, AIChE J. 51 (2005) 59-78.

[20] P.R. Gunjal, V.V. Ranade, R.V. Chaudhari, Experimental and computational study of liquid drop over flat and spherical surfaces, Catal. Today 79 (2003) 267-273.

[21] F. Schonfeld, S. Hardt, Dynamic contact angles in CFD simulations, Comput. Fluids 38 (2009) 757-764.

[22] H. Alla, S. Freifer, T. Roques-Carmes, A Computational fluid dynamics model using the volume of fluid method for describing the dynamics of spreading of Newtonian fluids, Colloids Surf. A 386 (2011) 107-115.

[23] T. Roques-Carmes, V. Mathieu, A. Gigante, Experimental contribution to the understanding of the dynamics of spreading of Newtonian fluids: Effect of volume, viscosity and surfactant, J. Colloid Interface Sci. 344 (2010) 180-197.

[24] K.N. Prabhu, P. Fernades, G. Kumar, Effect of substrate surface roughness on wetting behaviour of vegetable oils, Mater. Design 30 (2009) 297-305.

[25] A.M. Cazabat, M.A.C. Stuart, Dynamics of wetting: Effects of surface roughness, J. Phys. Chem. 90 (1986) 5845-5849.

[26] R. Scardovelli, S. Zaleski, Direct numerical simulation of free-surface and interfacial flow, Annu. Rev. Fluid Mech. 31 (1999) 567-603.

[27] S. Haeri, S.H. Hashemabadi, Three dimensional CFD simulation and experimental study of power law fluid spreading on inclined plates, Int. Commun. Heat Mass transfer 35 (2008) 1041-1047.

[28] L.S. Jang, G.H. Lin, Y.L. Lin, C.Y. Hsu, W.H. Kan, C.H. Chen, Simulation and experimentation of a microfluidics device based on electrowetting on dielectric, Biomed. Microdevices 9 (2007) 777-786.

[29] J.U. Brackbill, D.B. Kothe, C. Zemach, A continuum method for modeling surface tension, J. Comput. Phys. 100 (1992) 335-354.

[30] J. Li, Calcul d'interface affine par morceaux (Piecewise 


\section{Computational Fluid Dynamics}

linear interface calculation), C.R. Acad. Sci. II B 320 (1995) 391-396.

[31] M. Meier, G. Yadigaroglu, B.L. Smith, A novel technique for including surface tension in PLIC-VOF methods, Eur. J. Mech. B/Fluids 21 (2002) 61-73.

[32] S.F. Kistler, Hydrodynamics of Wetting, Wettability, Dekker, New York, 1993, pp. 311-429.

[33] K. Yokoi, D. Vadillo, J. Hinch, I. Hutchings, Numerical studies of the influence of the dynamic contact angle on a droplet impacting on a dry surface, Phys. Fluids 21 (2009) 072102-072112.

[34] S. Sikalo, H.D. Wilhelm, I.V. Roisman, S. Jakirlić, C. Tropea, Dynamic contact angle of spreading droplets: experiments and simulations, Phys. Fluids 17 (2005) 062103-062115.

[35] S. Afkhami, S. Zaleski, M. Bussmann, A mesh-dependent model for applying dynamic contact angles to VOF simulations, J. Comput. Phys. 228 (2009) 5370-5389.

[36] J. Bico, C. Tordeux, D. Quéré, Rough wetting, Europhys. Lett. 55 (2001) 214-220.

[37] H. Alla, S. Freifer, B.A. Talha, T. Roques-Carmes, New insight into the spreading dynamics of liquids on rough surfaces using computational fluid dynamics, Russ. Chem. Rev. 82 (2013) 1066-1080.
[38] F. Brochart-Wyart, J.M. di Meglio, D. Quéré, P.G. de Gennes, Spreading of non volatile liquids in a continuum picture, Langmuir 7 (1991) 335-338.

[39] L. Leger, J.F. Joanny, Liquid spreading, Rep. Prog. Phys. 55 (1992) 431-486.

[40] T. Roques-Carmes, V. Babak, V. Mathieu, A. Gigante, Influence of drop volume and surfactant concentration on the Marangoni flow and contact-line instabilities during the spreading of surfactant solutions on hydrophilic surface, J. Dispersion Sci. Technol. 34 (2013) 1053-1066.

[41] L. Bacri, G. Debrégeas, F. Brochard-Wyart, Experimental study of the spreading of a viscous droplet on a nonviscous liquid, Langmuir 12 (1996) 6708-6711.

[42] L. Bocquet, J.L. Barrat, Flow boundary conditions from nano- to micro-scales, Soft Matter 3 (2007) 685-693.

[43] Vinogradova, A. Belyaev, Wetting, roughness and flow boundary conditions, J. Phys.: Condens. Matter 23 (2011) 184104-184119.

[44] O.I. Vinogradova, A.L Dubov, Superhydrophobic textures for microfluidics, Mendeleev Commun. 22 (2012) 229-236

[45] A.M. Cazabat, M.A.Cohen Stuart, Dynamics of wetting on smooth and rough surfaces, Progr. Colloid \& Polymer Sci. 74 (1987) 69-75. 